\title{
Systemic Chemotherapy in Advanced Pancreatic Cancer
}

\author{
Hee Seung Lee and Seung Woo Park \\ Department of Internal Medicine, Institute of Gastroenterology, Yonsei University College of Medicine, Seoul, Korea
}

Pancreatic cancer remains one of the most lethal cancers. These patients often have multiple symptoms, and integrated supportive care is critical in helping them remain well for as long as possible. Fluorouracil-based chemotherapy is known to improve overall survival (OS) by approximately 3 months, compared to the best supportive care alone. A 1997 study comparing gemcitabine and fluorouracil treatment of advanced pancreatic cancer patients showed an improvement in OS of 1 month in patients receiving gemcitabine. Over the next 10 years, multiple randomized studies compared singleagent gemcitabine with combination chemotherapy and showed no effective survival improvement. However, the addition of erlotinib, an epidermal growth factor receptor (EGFR) inhibitor, was associated with a significant improvement in OS of approximately 2 weeks. However, adoption of this regimen has not been widespread because of its limited effect and added toxicity. Two clinical trials have recently prolonged OS in advanced pancreatic cancer patients by almost 1 year. The first compared FOLFIRINOX with gemcitabine alone, and was associated with a significant improvement in median survival. The second compared gemcitabine and nabpaclitaxel with gemcitabine alone, and was associated with improvements in OS. At present, these regimens are considered standard treatment for patients with good performance statuses. (Gut Liver 2016;10:340-347)

Key Words: Pancreatic neoplasms; Drug therapy; Gemcitabine; Fluorouracil

\section{INTRODUCTION}

The prognosis for pancreatic cancer patients is very poor. Patients with locally advanced disease have a median survival time of 8 to 12 months, and patients with distant metastasis have significantly worse outcomes, with a median survival time of only 3 to 6 months. ${ }^{1}$

Most patients (80\%) present with inoperable advanced pancreatic cancer at initial diagnosis; their management is a significant unmet challenge. Chemotherapy remains the mainstay of treatment for patients presenting with advanced pancreatic cancer, resulting in an increase in overall survival (OS). It is the eighth leading cause of death from cancer in men and the ninth leading cause of death from cancer in women throughout the world. ${ }^{1}$ In the Republic of Korea, approximately 5,400 people develop exocrine pancreatic cancer each year, and because of its aggressive character and the fact that most patients present with relatively advanced disease, most of them die from the disease. ${ }^{2}$

Recently completed phase III clinical trials have shown that an increasing number of chemotherapeutic options are now available for patients with advanced pancreatic cancer. These modern chemotherapy regimens are associated with a median survival approaching 1 year in selected patients with advanced pancreatic cancer, ${ }^{3}$ and we are now starting to think strategically about how to apply these different regimens to pancreatic cancer patients (Fig. 1).

Despite these advances in treatment options, the number of pancreatic cancer-related deaths continues to increase, and pancreatic cancer is expected to represent the second-leading cause of cancer-related mortality in the United States by the year 2020. ${ }^{4}$ This trend reflects the limitations of the current therapeutic regimens, the characteristics of pancreatic cancer (which is generally diagnosed at an advanced stage), and the absence of effective biomarkers. This article reviews the past and current systemic chemotherapy regimens used to treat patients with advanced pancreatic cancer. In addition, we will briefly discuss the issues of current alternative treatments, such as immunotherapy, epigenetic therapy, and stromal therapy, even though these are beyond the scope of our review.

Correspondence to: Seung Woo Park

Division of Gastroenterology, Department of Internal Medicine, Yonsei University College of Medicine, 50-1 Yonsei-ro, Seodaemun-gu, Seoul 03722, Korea

Tel: +82-2-2228-1964, Fax: +82-2-393-6884, E-mail: swoopark@yuhs.ac

Received on September 16, 2015. Accepted on December 1, 2015.

pISSN 1976-2283 eISSN 2005-1212 http://dx.doi.org/10.5009/gnl15465

@ This is an Open Access article distributed under the terms of the Creative Commons Attribution Non-Commercial License (http://creativecommons.org/licenses/by-nc/4.0) which permits unrestricted non-commercial use, distribution, and reproduction in any medium, provided the original work is properly cited. 


\section{CHEMOTHERAPY APPROACHES}

The primary goals of treatment for advanced pancreatic cancer are palliation and lengthened survival. At first, systemic chemotherapy treatment is recommended for patients with advanced pancreatic cancer and good performance statuses and is expected to result in prolonged survival. Depending on a patient's performance status, physicians can consider single agent or combination chemotherapy for this initial therapy. After cancer progression, second-line chemotherapy is also possible, especially in patients who maintain a good performance status. In the past, single agent chemotherapy has been the mainstay treatment for advanced pancreatic cancer. However, no single agent gave consistent median survival durations above 7 months (Table 1). And then, combination chemotherapy has become a new alternative in patients with advanced pancreatic cancer, with an increased survival benefit (Table 2).

\section{Fluorouracil}

Until 1997, fluorouracil (5-FU) was extensively studied using a variety of doses and schedules, but the response rate was very low (0\% to 10\%), there was no consistent effect on diseaserelated symptoms. ${ }^{5,6}$

\section{1) Capecitabine}

Capecitabine is a rationally designed oral fluoropyrimidine that is reliably absorbed intact through the intestinal wall and then converted to 5-FU. It is present at consistently higher levels in tumor rather than normal tissue, thereby providing the basis for enhanced selectivity and better tolerability. ${ }^{7}$ The efficacy of capecitabine monotherapy was shown in a study with advanced pancreatic cancer: ${ }^{8}$ three (7.3\%) of the 41 patients had an objective response and $10(24 \%)$ of the 41 patients experienced a clinical benefit response by improvement in pain intensity, analgesic consumption, and Karnofsky performance status.

\section{2) S-1}

S-1 is an oral fluoropyrimidine that includes three different agents (tegafur, gimeracil, and oteracil). S-1 monotherapy represents a reasonable alternative for patients who are not candidates for a more intensive first-line chemotherapy and who prefer the convenience of an oral regimen. In the phase III GEST trial of patients with locally advanced or metastatic pancreatic cancer, S-1 monotherapy was compared to either gemcitabine monotherapy or S-1 plus gemcitabine combination therapy. The OS in patients treated with S-1 were non-inferior to those with gemcitabine monotherapy (9.7 months vs 8.8 months, $\mathrm{p}<0.001$ for non-inferiority), and the objective response rate was significantly higher with S-1 treatment (21\% vs $13 \%)$.

\section{3) 5-FU plus doxorubicin and mitomycin (FAM)}

Despite essentially equivalent objective response rates and palliative effects (improved performance, body weight, or symptoms), randomized trials did not support a survival benefit for

Table 1. Randomized Controlled Trials of Monotherapy

\begin{tabular}{lccc}
\hline \multicolumn{1}{c}{ Regimen } & 5-FU & Gemcitabine & Capecitabine \\
\hline Study phase & III & III & II \\
Patients enrolled, n & 126 & 126 & 42 \\
Response rate, \% & 0 & 5.4 & 9.5 \\
Median survival, mo & 4.4 & 5.6 & 6.0 \\
Time to progression, mo & 0.9 & 2.1 & NR \\
Clinical benefit response, \% & 4.8 & 23.8 & 24.0 \\
\hline
\end{tabular}

5-FU, 5-fluorouracil; NR, not reported.

\begin{tabular}{|c|c}
1997 \\
5 -FU
\end{tabular}$\quad \begin{gathered}2007 \\
\text { Gemcitabine } \\
\text { erlotinib }\end{gathered} \quad \begin{gathered}2009 \\
\text { Gemcitabine+ } \\
\text { capecitabine }\end{gathered}$ FOLFIRINOX $\begin{gathered}2013 \\
\text { Gemcitabine+ } \\
\text { nab-paclitaxel }\end{gathered}$

Fig. 1. The trends of chemotherapeutic agents in advanced pancreatic cancer.

5-FU, 5-fluorouracil.

Table 2. Randomized Controlled Trials of Combination Chemotherapy Compared with Gemcitabine Monotherapy

\begin{tabular}{lcccc}
\hline \multicolumn{1}{c}{ Regimen } & Gemcitabine+erlotinib & Gemcitabine+capecitabine & FOLFIRINOX & Gemcitabine+nab-paclitaxel \\
\hline Year & 2007 & 2009 & 2011 & 2013 \\
Study phase & III & III & III & III \\
Patients enrolled, $\mathrm{n}$ & 569 & 533 & 31.6 vs 9.4 & 861 \\
Response rate, $\%$ & 8.6 vs 8.0 & 19.1 vs 12.4 & 11.1 vs 6.8 & 23.0 vs 7.0 \\
Median survival, mo & 6.3 vs 5.9 & 7.1 vs 6.2 & (p<0.001) & 8.5 vs 6.7 \\
& (p=0.038) & (p=0.08) & 6.4 vs 3.3 & $(\mathrm{p}<0.001)$ \\
Time to progression, mo & 3.7 vs 3.5 & 5.3 vs 3.8 & & 5.5 vs 3.7 \\
\hline
\end{tabular}


5-FU based combination regimens compared to 5-FU alone. Because of the failure to produce improved survival or palliation, the unrewarded toxicity, and the excessive cost, the FAM regimen was not recommended for the treatment of advanced pancreatic cancer. ${ }^{10}$

4) Methotrexate plus 5-FU, cyclophosphamide, vincristine, and mitomycin $\mathbf{C}$ (Mallinson regimen)

A phase III trial evaluated the Mallinson regimen and showed objective response rates of $21 \%$ and a median survival of 4.5 months, but this regimen was associated with significantly increased toxicity. Despite the higher objective response rates, this combination regimen did not give a survival advantage compared to 5-FU alone. ${ }^{11}$

\section{Gemcitabine}

In 1997, a clinical trial was performed to assess the effectiveness of gemcitabine in patients with newly diagnosed advanced pancreatic cancer. The trial randomly assigned 126 previously untreated patients with locally advanced or metastatic pancreatic cancer to 5 -FU or gemcitabine treatment. ${ }^{12}$ The primary efficacy measures of the trial were the clinical benefit response associated with pain (analgesic consumption and pain intensity), the Karnofsky performance status, and weight. Other measures of efficacy included the response rate, the time to progressive disease, and the survival. Gemcitabine was associated with significantly better clinical response $(24 \%$ vs $5 \%, p=0.0022)$ and median OS (5.6 months vs 4.4 months, $p=0.0025$ ). Based on these significant improvements in clinical benefit and survival, gemcitabine was approved as a first-line therapy for advanced pancreatic cancer.

\section{1) Gemcitabine plus 5-FU}

Gemcitabine has been combined with both bolus and infusional 5-FU. In a phase III trial, 5-FU and gemcitabine combined treatment did not improve the median survival of patients with advanced pancreatic carcinoma when compared with singleagent gemcitabine treatment (median survival, 6.7 months vs 5.4 months; $\mathrm{p}=0.09)$. $^{13}$

\section{2) Gemcitabine plus capecitabine}

For patients who want to gain some survival benefit from a less toxic regimen, gemcitabine plus capecitabine treatment may be an acceptable option. In 2007, a phase III trial compared the efficacy and safety of the combined gemcitabine plus capecitabine versus single-agent gemcitabine treatment in advanced pancreatic cancer. Median survival was not significantly improved by the addition of capecitabine in this trial (8.4 months vs 7.2 months, $\mathrm{p}=0.234) .{ }^{14}$ However, a second European trial found that progression-free survival (PFS) and objective response rates were significantly improved in patients receiving gemcitabine plus capecitabine when compared with gemcitabine treatment alone. The second trial also showed a trend towards an improvement in OS, even if it did not reach statistical significance (hazard ratio [HR], 0.86; 95\% confidence interval [CI], 0.72 to $1.02 ; \mathrm{p}=0.08) .{ }^{15}$ Moreover, a meta-analysis of the two phase III trials showed a significant survival benefit in favor of gemcitabine plus capecitabine (HR, 0.86; 95\% CI, 0.75 to 0.98 ; $\mathrm{p}=0.02$ ). The combination of gemcitabine plus capecitabine may be considered a standard first-line treatment option for advanced pancreatic cancer.

\section{3) Gemcitabine plus S-1}

In a phase III GEST trial, S-1 plus gemcitabine combined treatment was directly compared to gemcitabine monotherapy in patients with advanced pancreatic cancer. For the primary endpoint of OS, the combination (gemcitabine plus S-1) was not found to be superior to gemcitabine monotherapy (median, 10.1 months vs 8.8 months; $\mathrm{HR}, 0.88$; 95\% CI, 0.71 to $1.08 ; \mathrm{p}=0.15){ }^{9}{ }^{9}$

\section{4) Gemcitabine plus cisplatin}

Three phase III trials have compared gemcitabine treatment alone to the combination of gemcitabine plus cisplatin, ${ }^{16-18}$ but no significant survival advantage was achieved with this treatment approach (median survival, 7.9 months vs 7.7 months; $\mathrm{p}=0.857$ ). Nevertheless, selected patients with inherited forms of pancreatic cancer may have survival benefits when treated with platinum-based agent. ${ }^{19}$

\section{5) Gemcitabine plus irinotecan}

In 2006, a phase III trial was performed to determine the response rate and median and OS of gemcitabine as a monotherapy versus gemcitabine plus irinotecan combined treatment in advanced pancreatic cancer. No statistically significant difference was observed in this study with respect to OS (6.4 months vs 6.5 months). ${ }^{20}$

\section{6) Gemcitabine plus cisplatin, epirubicin and 5-FU (PEFG)}

In a randomized multicenter phase III trial, patients were randomly assigned to the PEFG regimen or gemcitabine monotherapy. The PEFG regimen was associated with a significantly higher response rate (39\% vs 9\%) and 4-month PFS (primary endpoint, 60\% vs 28\%). ${ }^{21}$ However, more patients in the PEFG group had grade 3 to 4 neutropenia (43\% vs 14\%) and thrombocytopenia (30\% vs 1\%) than patients in the gemcitabine monotherapy group $(\mathrm{p}<0.0001)$.

\section{7) Gemcitabine plus oxaliplatin (GEMOX)}

The efficacy of GEMOX was compared to gemcitabine treatment alone in two different multicenter trials of patients with advanced pancreatic cancer. In the first clinical trial, GEMOX was associated with significantly higher response rates (27\% vs $17 \%$ ) and significantly longer median PFS (5.8 months vs 3.7 months), but only a trend towards improved median sur- 
vival compared to gemcitabine monotherapy (9 months vs 7.1 months, $p=0.13) .^{22}$ In the second clinical trial of 832 patients with advanced pancreatic cancer, GEMOX did not show an improvement in median survival or any symptom benefit over standard gemcitabine monotherapy (5.7 months vs 4.9 months, $\mathrm{p}=0.22)^{23}$ There were also no significant benefits for GEMOX treatment in terms of objective response rate ( $9 \%$ vs $6 \%$ ) or PFS.

\section{8) Gemcitabine plus erlotinib}

Since 1997, gemcitabine has been combined with many other cytotoxic agents including 5-FU, cisplatin, docetaxel, oxaliplatin, and irinotecan. Despite encouraging early phase data, phase III randomized trials of such combinations have not demonstrated a statistically significant survival benefit compared to treatment with gemcitabine alone.

On the other hand, pancreatic cancers often express receptors for epidermal growth factor (EGF). A phase III trial directly compared gemcitabine treatment with and without erlotinib (an inhibitor of epidermal growth factor receptor [EGFR] tyrosine kinase) in patients with advanced pancreatic cancer. ${ }^{24}$ Combined therapy showed a statistically significant improvement in OS compared to gemcitabine treatment alone (HR, 0.82; $\mathrm{p}=0.038$; median, 6.2 months vs 5.9 months, respectively). PFS was also significantly longer with gemcitabine plus erlotinib combined treatment, with an estimated HR of 0.77 (95\% CI, 0.64 to 0.92; $\mathrm{p}=0.004)$.

Erlotinib was eventually approved for use in the United States for the treatment of advanced pancreatic cancer in combination with gemcitabine. However, there were some limitations. The combination of gemcitabine plus erlotinib treatment resulted in just a 2-week improvement in survival over gemcitabine treatment alone. Furthermore, the high percentage of activating KRAS mutations, which occur in up to $90 \%$ of pancreatic cancer patients, means that the pharmacological inhibition of EGFR upstream of KRAS remains only marginally effective in this cancer type. $^{25}$

\section{Taxanes}

Taxanes have shown limited benefit as monotherapies in advanced pancreatic cancer. In one phase II study, in which 33 patients with advanced pancreatic cancer received single agent docetaxel treatment along with granulocyte colony stimulating factor, the objective response rate was only $6 \%$. However, a disease-stabilizing effect was suggested because the 1-year survival was 36\% with clinical benefit. ${ }^{26}$ However, other studies have reported limited activity of taxanes as single agents against pancreatic cancer.

\section{Irinotecan}

Irinotecan has limited activity as a monotherapy in advanced pancreatic cancer, with low response rates of 9\% and median survival duration of 5.2 months. ${ }^{27}$ Topotecan has showed a total response rate of $8 \%$ and stable disease was seen in three patients. The median survival was 20 weeks. The time to progression and the median survival reported are similar to those observed with gemcitabine monotherapy in a randomized trial, suggesting that topotecan also has limited activity in advanced pancreatic cancer. $^{28}$

\section{TWO RECENT CLINICAL TRIALS SUPERIOR TO GEMCITABINE ALONE}

\section{The FOLFIRINOX regimen}

Newer regimens including 5-FU/leucovorin, oxaliplatin, and irinotecan (FOLFIRINOX) showed promising results for advanced pancreatic cancer. The randomized phase III trial evaluated FOLFIRINOX treatment versus gemcitabine monotherapy in chemotherapy-naïve patients with advanced pancreatic cancer, good performance statuses, and serum bilirubin $<1.5$ times the upper limit of normal. The results showed dramatic improvements in both median PFS (6.4 months vs 3.3 months, $\mathrm{p}<0.001$ ) and median OS (11.1 months vs 6.8 months, $\mathrm{p}<0.001){ }^{3}$ The trial was stopped after enrolling only 250 patients, when a preplanned interim analysis demonstrated that the primary trial endpoint (improved OS) had been met.

Treatment-related toxicity was, however, also significantly worse with FOLFIRINOX treatment, including grade 3/4 neutropenia (45.7\%), thrombocytopenia (9.1\%), and diarrhea (12.7\%). These data eventually established FOLFIRINOX as the preferred first-line therapy for patients with advanced pancreatic cancer who have a good performance status.

\section{Gemcitabine plus nab-paclitaxel}

Gemcitabine plus nanoparticle albumin bound (nab)-paclitaxel represents an acceptable alternative to FOLFIRINOX. Von Hoff et al. ${ }^{29}$ reported promising results from the Metastatic Pancreatic Adenocarcinoma Clinical Trial (MPACT), an international phase III study in which 861 patients were randomly assigned to receive either gemcitabine treatment alone or the combination of gemcitabine plus nab-paclitaxel. The addition of this taxane agent resulted in a significant improvement in OS (8.5 months vs 6.7 months; $\mathrm{p}<0.001$; HR, 0.72; 95\% CI, 0.62 to 0.83), with a manageable and predictable toxicity profile. Grade 3/4 adverse events, that were seen more often with this combination therapy, included neutropenia ( $38 \%$ vs $27 \%$ ), febrile neutropenia (3\% vs 1\%), and diarrhea (6\% vs 1\%). Some studies investigated the biological effects of this regimen and suggested that tumors treated with nab-paclitaxel contained fewer cancer associated fibroblasts and a clearly altered collagen that results in tumor softening at the tissue level. ${ }^{29}$

In addition to the hypothesis that the tumor cells themselves are albumin-avid leading to enhanced tumoral concentration, it has been hypothesized that osteonectin-a secreted protein that is acidic and rich in cysteine, and which is highly expressed and 
secreted by pancreatic peritumoral fibroblasts-may serve as an albumin-binding protein that sequesters nab-paclitaxel and concentrates the drug intratumorally. ${ }^{30}$

\section{SECOND-LINE SYSTEMIC CHEMOTHERAPY}

For patients who are refractory to a gemcitabine-containing regimen, a limited amount of data suggest that a second-line chemotherapy may improve survival and it is, therefore, reasonable to consider further chemotherapy in patients who maintain a good performance status. For patients with advanced disease who have received prior gemcitabine-based therapy, fluoropyrimidine-based chemotherapy regimens are acceptable secondline options. Alternatively, gemcitabine-based therapy can be given to those previously treated with fluoropyrimidine-based therapy. However, there are no widely accepted optimal regimens for second-line therapy.

There are few randomized trials of second-line chemotherapy for patients who have failed a gemcitabine-based chemotherapy. A phase II trial was conducted to explore the efficacy of capecitabine and oxaliplatin (XELOX) in patients with advanced pancreatic cancer who were previously treated with gemcitabine. The Kaplan-Meier estimate of the overall median survival was 23 weeks (95\% CI, 17.0 to 31.0 weeks). The XELOX regimen is active in gemcitabine-pretreated patients with advanced pancreatic cancer, especially in patients with a good performance status and those who have responded to first-line chemotherapy. ${ }^{31}$

A phase III trial comparing the OFF regimen (oxaliplatin, 5-FU, and folinic acid) to best supportive care provided firsttime evidence for the benefit of second-line chemotherapy in advanced pancreatic cancer, manifested by prolonged secondline survival (4.8 months vs 2.3 months, $\mathrm{p}=0.008$ ) and median OS (9.1 months vs 7.9 months, $\mathrm{p}=0.031)$. $^{32}$ The AIO-PK0104 trial also assessed second-line therapy in a randomized crossover trial and found capecitabine to be efficacious after progression in patients with advanced disease on gemcitabine and erlotinib combined primary treatment. ${ }^{33}$ On the other hand, the optimal second-line chemotherapy for patients who fail initial FOLFIRINOX treatment is undefined. For those patients who retain a good performance status, gemcitabine monotherapy may be a reasonable option.

\section{MOLECULAR TARGETED THERAPY}

\section{KRAS}

New systemic therapies have been designed based upon the biological properties of pancreatic cancer. The KRAS oncogene has been the subject of considerable research over the last decade. KRAS proteins are membrane-bound GTP-binding proteins that act as molecular switches in downstream signal transduction. ${ }^{34}$ Mutations of the KRAS gene, present in ap- proximately $90 \%$ of pancreatic cancers, lead to the constitutive activation of these proteins and tumorigenesis. ${ }^{35}$

In order to become active, KRAS proteins require the posttranslational addition of a 15-carbon farnesyl group. If the addition of this farnesyl group is blocked, the protein cannot attach to the cell membrane and the protein remains inactive. Farnesyl transferase inhibitors were designed to prevent membrane association and thereby activation of KRAS. However, the oral farnesyl transferase inhibitor R115777 did not increase the median survival time of patients with advanced pancreatic cancer (112 days vs 109 days, $p=0.75$ ). It is likely that nonfarnesylated KRAS can still undergo prenylation, via geranylgeranylation, to associate with the cell membrane. ${ }^{34,36}$

\section{EGFR and VEGF}

As noted above, the combination of gemcitabine plus erlotinib resulted in a modest 2-week improvement in survival over gemcitabine treatment alone in a phase III trial. Since then, numerous EGFR or vascular endothelial growth factor (VEGF) targeted agents have been evaluated, alone or in combination with chemotherapy, in advanced pancreatic cancer. Unfortunately, most agents have failed to improve patient survival significantly. The list of agents tested in trials include antiangiogenic drugs, such as bevazicumab; monoclonal antibodies directed against EGFR, such as cetuximab; and multikinase inhibitors with antiangiogenic activity, such as axitinib, sunitinib, and sorafenib. $^{37-41}$

\section{EMERGING CONCEPTS}

\section{Stromal therapy}

The extensive desmoplastic reaction that comprises up to $90 \%$ of the tumor volume is the predominant histological feature of pancreatic cancer. Inefficient drug delivery due to the intense stromal reaction may be an important contributor to chemoresistance in pancreatic cancer. ${ }^{42}$ The inhibition of stroma-related signaling pathways is considered to be a promising tool to decrease stromal density and to facilitate the access of cytotoxic drugs to the tumor cells.

The sonic hedgehog pathway is one of the predominant signaling cascades known to stimulate the stromal reaction. Treatment with the hedgehog inhibitor IPI926 resulted in a significant depletion of tumor-associated stroma. However, the interim analysis of a clinical trial found that the OS of patients on the gemcitabine plus IPI926 treatment arm was inferior to that of patients treated with gemcitabine plus placebo. On the other hand, clinical trials for other hedgehog inhibitors, such as GDC-0449 in combination with gemcitabine or LDE225 in combination with FOLFIRINOX or gemcitabine, are still ongoing (NCT01088815, NCT02358161).

The transforming growth factor $\beta$ (TGF- $\beta$ )-dependent signaling cascade is known to be another key pathway implicated 
in the stromal reaction which is associated with invasion, metastasis, angiogenesis, and escape from immune-surveillance in pancreatic cancer. ${ }^{43,44}$ A phase I/II trial is currently ongoing to test the impact of LY2157299-a small molecule that has been designed to selectively inhibit the TGF $\beta$ type I receptorin combination with gemcitabine treatment for patients with advanced pancreatic cancer (NCT01373164). Acellular matrix components are currently being evaluated as targets for therapeutic intervention. Human recombinant PH20 hyaluronidase (PEGPH20) enzymatically depletes hyaluronan (highly abundant in the extracellular matrix of both human and murine pancreatic cancer tissues), thereby inducing the re-expansion of tumor blood vessels and increasing the concentration of gemcitabine within the tumor. ${ }^{45}$ PEGPH20 resulted in significantly diminished tumor growth and prolonged survival in mice. A phase II trial comparing PEGPH20 treatment combined with gemcitabine plus nab-paclitaxel versus gemcitabine plus nab-paclitaxel treatment alone is currently recruiting to evaluate the impact of depleting hyaluronan in patients with advanced pancreatic cancer (NCT01839487).

\section{Immunotherapy}

Immunotherapy stimulates a host immune response that results in long-term tumor destruction. The stroma of pancreatic cancer is particularly rich in inflammatory cells that are proposed to mediate drug resistance and tumor progression. Therefore, immune cells-such as T-cells and macrophagesinfiltrating the peri-tumoral stroma represent a promising target for immunotherapeutic approaches. ${ }^{46,47}$

T-cell mediated immunity includes multiple sequential steps that are regulated by counterbalancing stimulatory and inhibitory signals that fine-tune the response. These inhibitory pathways are referred to as immune checkpoints and these are crucial for maintaining self-tolerance and modulating the duration and amplitude of physiological immune responses. Cytotoxic T-lymphocyte-associated antigen 4 (CTLA-4) is one of these immune checkpoints that plays a critical role in regulating and limiting immune responses, and it can be blocked by specific antibodies such as ipilimumab, a fully human antibody. The binding of ipilimumab to CTLA-4 blocks the immune limiting activity of CTLA-4, thereby sustaining an active immune response against the cancer cells. Early phase trials are currently underway in pancreatic cancer for ipilimumab (NCT01473940).

Another promising immunomodulatory target is CD40, which is a costimulatory molecule for antigen presenting cells. Gemcitabine treatment combined with CD40 agonist-activated Tcells reduced the tumor burden in advanced pancreatic cancer patients in a phase I study by decreasing tumor stroma and increasing the tumor infiltration of activated macrophages. ${ }^{48}$

In addition to enhancing the systemic immune response, attracting selected antitumor cytokines is also a promising concept that is currently under intense preclinical and clinical investigation. One of the most tumor selective antigens that can be used to guide cytokines to the site of a tumor is the extradomain B (ED-B) of fibronectin. The fusion product consisting of the ED-B antibody fragment L19 and interleukin-2 is currently being tested in a phase I/II trial in pancreatic cancer (NCT01198522).

\section{Histone deacetylase inhibitor}

Chromatin is formed by the wrapping of DNA around histones, a process that is regulated by histone acetylation status. The acetylation of histones removes their positive charge, leading to a more relaxed chromatin structure that is associated with transcriptionally active DNA. This relaxation can be reversed by histone deacetylases (HDAC). Epigenetic regulation of tumor suppressor genes via HDAC is involved in the apoptosis, differentiation, and growth of cells, all of which influence tumor cell survival. HDAC inhibitors, such as vorinostat, have been developed and have demonstrated significant antitumor activity in a variety of malignancies. In pancreatic cancer, vorinostat is currently being evaluated in combination with radiation therapy and chemotherapy in phase I/II clinical trials (NCT00243100, NCT00978688).

\section{Telomerase peptide vaccine}

Telomerase is a ribonucleotide enzyme that maintains cellular stability and is expressed by almost all cancer cells. ${ }^{49} \mathrm{~A}$ phase III trial was performed to assess the efficacy and safety of telomerase peptide vaccine (GV1001) in combination with chemotherapy (gemcitabine and capecitabine) treatment in patients with advanced pancreatic cancer. However, adding GV1001 vaccination to chemotherapy (gemcitabine and capecitabine) treatment did not improve OS in these patients.

Despite the disappointing phase III results, the findings have identified biomarkers that may predict response to this vaccine and new research may indicate benefits in a subgroup of patients. Baseline eotaxin levels were measured in patients receiving concurrent chemoimmunotherapy. High eotaxin levels may predict an improved survival in patients receiving GV1001 with gemcitabine and capecitabine treatment (high eotaxin, 14.8 months; low eotaxin, 7.9 months; $p=0.0135$ ). There is another ongoing study with chemoradiotherapy in patients with locally advanced pancreatic cancer (NCT01342224). ${ }^{50}$

\section{CONCLUSIONS}

Despite multiple clinical trials and continuous efforts, pancreatic cancer remains one of the most difficult cancers to cure, both because of its resistance to conventional chemotherapy and because of its aggressive characteristics. Recent clinical trials have shown that newly combined chemotherapies can prolong OS in advanced pancreatic cancer. ${ }^{3,51}$ These therapeutic advances are more likely to produce gradual improvements 
in patient outcomes, rather than innovative change. Although conventional chemotherapy remains the standard of care for advanced pancreatic cancer, a variety of novel therapeutic approaches-including signal transduction inhibitors, immunotherapy, stromal depleting agents, and epigenetic therapyare currently under active investigation. Several studies have already demonstrated encouraging results in phase I/II studies. In the future, these new insights into the biology and genetics of pancreatic cancer may be of value in the development of new chemotherapeutic regimens.

\section{CONFLICTS OF INTEREST}

No potential conflict of interest relevant to this article are reported.

\section{REFERENCES}

1. Ryan DP, Hong TS, Bardeesy N. Pancreatic adenocarcinoma. N Engl J Med 2014;371:1039-1049.

2. National Cancer Center. National cancer registration program [Internet]. Goyang: National Cancer Center; 2012 [cited 2015 Aug 10]. Available from: https://ncc.re.kr/main.ncc?uri=english/sub04_ ControlPrograms02.

3. Conroy T, Desseigne F, Ychou M, et al. FOLFIRINOX versus gemcitabine for metastatic pancreatic cancer. N Engl J Med 2011;364: 1817-1825.

4. Ko AH. Progress in the treatment of metastatic pancreatic cancer and the search for next opportunities. J Clin Oncol 2015;33:1779-1786.

5. DeCaprio JA, Mayer RJ, Gonin R, Arbuck SG. Fluorouracil and high-dose leucovorin in previously untreated patients with advanced adenocarcinoma of the pancreas: results of a phase II trial. J Clin Oncol 1991;9:2128-2133.

6. Van Rijswijk RE, Jeziorski K, Wagener DJ, et al. Weekly high-dose 5-fluorouracil and folinic acid in metastatic pancreatic carcinoma: a phase II study of the EORTC GastroIntestinal Tract Cancer Cooperative Group. Eur J Cancer 2004;40:2077-2081.

7. Schüller J, Cassidy J, Dumont E, et al. Preferential activation of capecitabine in tumor following oral administration to colorectal cancer patients. Cancer Chemother Pharmacol 2000;45:291-297.

8. Cartwright TH, Cohn A, Varkey JA, et al. Phase II study of oral capecitabine in patients with advanced or metastatic pancreatic cancer. J Clin Oncol 2002;20:160-164.

9. Ueno H, Ioka T, Ikeda M, et al. Randomized phase III study of gemcitabine plus S-1, S-1 alone, or gemcitabine alone in patients with locally advanced and metastatic pancreatic cancer in Japan and Taiwan: GEST study. J Clin Oncol 2013;31:1640-1648.

10. Cullinan SA, Moertel CG, Fleming TR, et al. A comparison of three chemotherapeutic regimens in the treatment of advanced pancreatic and gastric carcinoma: fluorouracil vs fluorouracil and doxorubicin vs fluorouracil, doxorubicin, and mitomycin. JAMA 1985;253:2061-2067.
11. Cullinan S, Moertel CG, Wieand HS, et al. A phase III trial on the therapy of advanced pancreatic carcinoma: evaluations of the Mallinson regimen and combined 5-fluorouracil, doxorubicin, and cisplatin. Cancer 1990;65:2207-2212.

12. Burris HA 3rd, Moore MJ, Andersen J, et al. Improvements in survival and clinical benefit with gemcitabine as first-line therapy for patients with advanced pancreas cancer: a randomized trial. J Clin Oncol 1997;15:2403-2413.

13. Berlin JD, Catalano P, Thomas JP, Kugler JW, Haller DG, Benson $A B$ 3rd. Phase III study of gemcitabine in combination with fluorouracil versus gemcitabine alone in patients with advanced pancreatic carcinoma: Eastern Cooperative Oncology Group Trial E2297. J Clin Oncol 2002;20:3270-3275.

14. Herrmann R, Bodoky G, Ruhstaller T, et al. Gemcitabine plus capecitabine compared with gemcitabine alone in advanced pancreatic cancer: a randomized, multicenter, phase III trial of the Swiss Group for Clinical Cancer Research and the Central European Cooperative Oncology Group. J Clin Oncol 2007;25:22122217.

15. Cunningham D, Chau I, Stocken DD, et al. Phase III randomized comparison of gemcitabine versus gemcitabine plus capecitabine in patients with advanced pancreatic cancer. J Clin Oncol 2009;27: 5513-5518.

16. Heinemann V, Quietzsch D, Gieseler F, et al. Randomized phase III trial of gemcitabine plus cisplatin compared with gemcitabine alone in advanced pancreatic cancer. J Clin Oncol 2006;24:3946-3952.

17. Colucci G, Labianca R, Di Costanzo F, et al. Randomized phase III trial of gemcitabine plus cisplatin compared with single-agent gemcitabine as first-line treatment of patients with advanced pancreatic cancer: the GIP-1 study. J Clin Oncol 2010;28:1645-1651.

18. Chao Y, Wu CY, Wang JP, Lee RC, Lee WP, Li CP. A randomized controlled trial of gemcitabine plus cisplatin versus gemcitabine alone in the treatment of metastatic pancreatic cancer. Cancer Chemother Pharmacol 2013;72:637-642.

19. Ferrone CR, Levine DA, Tang LH, et al. BRCA germline mutations in Jewish patients with pancreatic adenocarcinoma. J Clin Oncol 2009;27:433-438.

20. Stathopoulos GP, Syrigos K, Aravantinos G, et al. A multicenter phase III trial comparing irinotecan-gemcitabine (IG) with gemcitabine $(\mathrm{G})$ monotherapy as first-line treatment in patients with locally advanced or metastatic pancreatic cancer. Br J Cancer 2006;95:587-592.

21. Reni M, Cordio S, Milandri C, et al. Gemcitabine versus cisplatin, epirubicin, fluorouracil, and gemcitabine in advanced pancreatic cancer: a randomized controlled multicentre phase III trial. Lancet Oncol 2005;6:369-376.

22. Louvet C, Labianca R, Hammel P, et al. Gemcitabine in combination with oxaliplatin compared with gemcitabine alone in locally advanced or metastatic pancreatic cancer: results of a GERCOR and GISCAD phase III trial. J Clin Oncol 2005;23:3509-3516.

23. Poplin E, Feng Y, Berlin J, et al. Phase III, randomized study of gemcitabine and oxaliplatin versus gemcitabine (fixed-dose rate 
infusion) compared with gemcitabine (30-minute infusion) in patients with pancreatic carcinoma E6201: a trial of the Eastern Cooperative Oncology Group. J Clin Oncol 2009;27:3778-3785.

24. Moore MJ, Goldstein D, Hamm J, et al. Erlotinib plus gemcitabine compared with gemcitabine alone in patients with advanced pancreatic cancer: a phase III trial of the National Cancer Institute of Canada Clinical Trials Group. J Clin Oncol 2007;25:1960-1966.

25. Michl P, Gress TM. Current concepts and novel targets in advanced pancreatic cancer. Gut 2013;62:317-326.

26. Androulakis N, Kourousis C, Dimopoulos MA, et al. Treatment of pancreatic cancer with docetaxel and granulocyte colonystimulating factor: a multicenter phase II study. J Clin Oncol 1999;17:1779-1785.

27. Wagener DJ, Verdonk HE, Dirix LY, et al. Phase II trial of CPT11 in patients with advanced pancreatic cancer, an EORTC early clinical trials group study. Ann Oncol 1995;6:129-132.

28. Stevenson JP, Scher RM, Kosierowski R, et al. Phase II trial of topotecan as a 21-day continuous infusion in patients with advanced or metastatic adenocarcinoma of the pancreas. Eur J Cancer 1998;34:1358-1362.

29. Von Hoff DD, Ervin T, Arena FP, et al. Increased survival in pancreatic cancer with nab-paclitaxel plus gemcitabine. N Engl J Med 2013;369:1691-1703.

30. Infante JR, Matsubayashi H, Sato N, et al. Peritumoral fibroblast SPARC expression and patient outcome with resectable pancreatic adenocarcinoma. J Clin Oncol 2007;25:319-325.

31. Xiong HQ, Varadhachary GR, Blais JC, Hess KR, Abbruzzese JL, Wolff RA. Phase 2 trial of oxaliplatin plus capecitabine (XELOX) as second-line therapy for patients with advanced pancreatic cancer. Cancer 2008;113:2046-2052.

32. Pelzer U, Schwaner I, Stieler J, et al. Best supportive care (BSC) versus oxaliplatin, folinic acid and 5-fluorouracil (OFF) plus BSC in patients for second-line advanced pancreatic cancer: a phase III-study from the German CONKO-study group. Eur J Cancer 2011;47:1676-1681.

33. Heinemann V, Vehling-Kaiser U, Waldschmidt D, et al. Gemcitabine plus erlotinib followed by capecitabine versus capecitabine plus erlotinib followed by gemcitabine in advanced pancreatic cancer: final results of a randomised phase 3 trial of the 'Arbeitsgemeinschaft Internistische Onkologie' (AIO-PK0104). Gut 2013;62:751-759.

34. di Magliano MP, Logsdon CD. Roles for KRAS in pancreatic tumor development and progression. Gastroenterology 2013;144:12201229.

35. Bryant KL, Mancias JD, Kimmelman AC, Der CJ. KRAS: feeding pancreatic cancer proliferation. Trends Biochem Sci 2014;39:91100.

36. Van Cutsem E, van de Velde H, Karasek P, et al. Phase III trial of gemcitabine plus tipifarnib compared with gemcitabine plus placebo in advanced pancreatic cancer. J Clin Oncol 2004;22:1430-1438.

37. Kindler HL, Niedzwiecki D, Hollis D, et al. Gemcitabine plus bevacizumab compared with gemcitabine plus placebo in patients with advanced pancreatic cancer: phase III trial of the Cancer and Leukemia Group B (CALGB 80303). J Clin Oncol 2010;28:3617-3622.

38. Kindler HL, Wroblewski K, Wallace JA, et al. Gemcitabine plus sorafenib in patients with advanced pancreatic cancer: a phase II trial of the University of Chicago Phase II Consortium. Invest New Drugs 2012;30:382-386.

39. O'Reilly EM, Niedzwiecki D, Hall M, et al. A Cancer and Leukemia Group B phase II study of sunitinib malate in patients with previously treated metastatic pancreatic adenocarcinoma (CALGB 80603). Oncologist 2010;15:1310-1319.

40. Van Cutsem E, Vervenne WL, Bennouna J, et al. Phase III trial of bevacizumab in combination with gemcitabine and erlotinib in patients with metastatic pancreatic cancer. J Clin Oncol 2009;27: 2231-2237.

41. Philip PA, Benedetti J, Corless CL, et al. Phase III study comparing gemcitabine plus cetuximab versus gemcitabine in patients with advanced pancreatic adenocarcinoma: Southwest Oncology Group-directed intergroup trial S0205. J Clin Oncol 2010;28:36053610.

42. Olive KP, Jacobetz MA, Davidson CJ, et al. Inhibition of Hedgehog signaling enhances delivery of chemotherapy in a mouse model of pancreatic cancer. Science 2009;324:1457-1461.

43. Hilbig A, Oettle H. Transforming growth factor beta in pancreatic cancer. Curr Pharm Biotechnol 2011;12:2158-2164.

44. Fuxe J, Karlsson MC. TGF-beta-induced epithelial-mesenchymal transition: a link between cancer and inflammation. Semin Cancer Biol 2012;22:455-461.

45. Jacobetz MA, Chan DS, Neesse A, et al. Hyaluronan impairs vascular function and drug delivery in a mouse model of pancreatic cancer. Gut 2013;62:112-120.

46. Vanneman M, Dranoff G. Combining immunotherapy and targeted therapies in cancer treatment. Nat Rev Cancer 2012;12:237251.

47. Dodson LF, Hawkins WG, Goedegebuure P. Potential targets for pancreatic cancer immunotherapeutics. Immunotherapy 2011;3: 517-537.

48. Beatty GL, Chiorean EG, Fishman MP, et al. CD40 agonists alter tumor stroma and show efficacy against pancreatic carcinoma in mice and humans. Science 2011;331:1612-1616.

49. Vasef MA, Ross JS, Cohen MB. Telomerase activity in human solid tumors: diagnostic utility and clinical applications. Am J Clin Pathol 1999;112(1 Suppl 1):S68-S75.

50. Middleton G, Silcocks P, Cox T, et al. Gemcitabine and capecitabine with or without telomerase peptide vaccine GV1001 in patients with locally advanced or metastatic pancreatic cancer (TeloVac): an open-label, randomised, phase 3 trial. Lancet Oncol 2014;15:829-840.

51. Park S, Chung MJ, Park JY, et al. Phase II trial of erlotinib plus gemcitabine chemotherapy in Korean patients with advanced pancreatic cancer and prognostic factors for chemotherapeutic response. Gut Liver 2013;7:611-615. 